\title{
Article \\ Optical Constants of Chemical Vapor Deposited Graphene for Photonic Applications
}

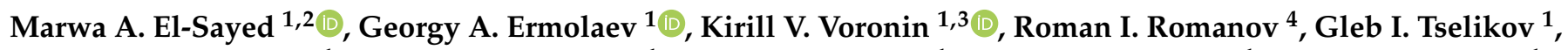 \\ Dmitry I. Yakubovsky ${ }^{1}$, Natalia V. Doroshina ${ }^{1}$, Anton B. Nemtsov ${ }^{1}$, Valentin R. Solovey ${ }^{1}$, Artem A. Voronov ${ }^{1}$, \\ Sergey M. Novikov ${ }^{1}{ }^{1}$, Andrey A. Vyshnevyy ${ }^{1}{ }^{1}$, Andrey M. Markeev ${ }^{1}$, Aleksey V. Arsenin $\left.{ }^{1,5}{ }^{(}\right)$ \\ and Valentyn $S$. Volkov $1,5, *$ (i)
}

check for updates

Citation: El-Sayed, M.A.; Ermolaev, G.A.; Voronin, K.V.; Romanov, R.I.; Tselikov, G.I.; Yakubovsky, D.I.; Doroshina, N.V.; Nemtsov, A.B.; Solovey, V.R.; Voronov, A.A.; et al. Optical Constants of Chemical Vapor Deposited Graphene for Photonic Applications. Nanomaterials 2021, 11, 1230. https://doi.org/10.3390/ nano11051230

Academic Editor: Gwan-Hyoung Lee

Received: 12 April 2021

Accepted: 25 April 2021

Published: 7 May 2021

Publisher's Note: MDPI stays neutral with regard to jurisdictional claims in published maps and institutional affiliations.

Copyright: (c) 2021 by the authors. Licensee MDPI, Basel, Switzerland. This article is an open access article distributed under the terms and conditions of the Creative Commons Attribution (CC BY) license (https:/ / creativecommons.org/licenses/by/ $4.0 /)$.
1 Center for Photonics and 2D Materials, Moscow Institute of Physics and Technology, 9 Institutsky Lane, 141700 Dolgoprudny, Russia; mira@phystech.edu (M.A.E.-S.); georgiy.ermolayev@phystech.edu (G.A.E.); voronin.kv@phystech.edu (K.V.V.); tselikov.gi@mipt.ru (G.I.T.); dmitrii.yakubovskii@phystech.edu (D.I.Y.); doroshina.nv@phystech.edu (N.V.D.); nemtsov@phystech.edu (A.B.N.); solovey.vr@phystech.edu (V.R.S.); voronov.aa@mipt.ru (A.A.V.); novikov.s@mipt.ru (S.M.N.); andrey.vyshnevyy@phystech.edu (A.A.V.); markeev.am@mipt.ru (A.M.M.); arsenin.av@mipt.ru (A.V.A.)

2 Department of Physics, Faculty of Science, Menoufia University, Shebin El-Koom 32511, Egypt

3 Skolkovo Institute of Science and Technology, 3 Nobel, 143026 Moscow, Russia

4 Moscow Engineering Physics Institute, National Research Nuclear University MEPhI, 31 Kashirskoe Sh., 115409 Moscow, Russia; limpo2003@mail.ru

5 GrapheneTek, Skolkovo Innovation Center, 143026 Moscow, Russia

* Correspondence: volkov.vs@mipt.ru; Tel.: +7-926-735-93-98

\begin{abstract}
Graphene is a promising building block material for developing novel photonic and optoelectronic devices. Here, we report a comprehensive experimental study of chemical-vapor deposited (CVD) monolayer graphene's optical properties on three different substrates for ultraviolet, visible, and near-infrared spectral ranges (from 240 to $1000 \mathrm{~nm}$ ). Importantly, our ellipsometric measurements are free from the assumptions of additional nanometer-thick layers of water or other media. This issue is critical for practical applications since otherwise, these additional layers must be included in the design models of various graphene photonic, plasmonic, and optoelectronic devices. We observe a slight difference (not exceeding $5 \%$ ) in the optical constants of graphene on different substrates. Further, the optical constants reported here are very close to those of graphite, which hints on their applicability to multilayer graphene structures. This work provides reliable data on monolayer graphene's optical properties, which should be useful for modeling and designing photonic devices with graphene.
\end{abstract}

Keywords: graphene; optical constants; dielectric properties; refractive index; nanophotonics; spectroscopic ellipsometry

\section{Introduction}

Graphene is one of the most attractive materials for the development of promising new photonic, plasmonic, and optoelectronic devices [1-7]. In particular, graphene is a critical component of nanoscale broadband optical modulators [8-10], chip-integrated ultrafast photodetectors [11], highly sensitive and selective sensors [12,13], transparent, flexible solar cells [14,15], among others. The design, simulation, and optimization of such functional components and devices all require knowledge of graphene's optical constants. A precise method for determining refractive indices and extinction coefficients is spectroscopic ellipsometry, which allows for extracting the dielectric function in a broad wavelength range directly from the raw data $[16,17]$. The previous works report ellipsometric studies of the optical constants of exfoliated graphene [18-22], epitaxial graphene [23-25], and CVD graphene [26-31], also transferred onto various substrates such as optical glass [26], silicon oxide $[18,20,31]$, or fused silica $[18,19,29,30]$. However, the measured dielectric functions 
show more than $20 \%$ differences, caused not only by the graphene production technique, the effect of the substrate, or the quality of graphene but also by the use of different ellipsometric models and respective initial assumptions. For instance, in the analysis of optical properties, one can take into account the adsorption of water $[18,28,30-32]$ and polymer residues $[18,19]$ on graphene used for the transfer of both CVD and exfoliated graphene. Therefore, despite extensive research efforts devoted to studying monolayer graphene's optical response, optical constants' choice remains challenging due to the lack of consensus in the field.

It is well-known that graphene is easily contaminated by adsorbing water and other organic and inorganic compounds [33,34], but, in most cases, it is meticulously cleaned from contamination and protected from the external environment by encapsulation layers [35]. However, even with thorough cleaning and annealing of graphene from contamination, trace amounts of water or organic molecules may remain. One way to overcome this issue is to include residual contaminants as additional layers in an ellipsometric model to obtain the optical properties of pure graphene. Yet, this approach has its drawbacks. Firstly, one should know the exact amount of contamination, which is very difficult to determine. Furthermore, even if the amount of residues is determined, it is not guaranteed that their influence on graphene sample's properties can be properly taken into account by adding auxiliary planar layers. Secondly, to assess the expected performance of graphene-based photonic and optoelectronic devices, one should not forget to include these contamination layers in the device model. Additionally, to evaluate the maximum performance of devices, it is essential to investigate the dielectric response of cleaned graphene on typical substrates.

Here, we present the optical properties of commercially available monolayer CVD graphene on glass, quartz, and $\mathrm{SiO}_{2} / \mathrm{Si}$ substrates. The optical properties were investigated by highly accurate variable-angle spectroscopic ellipsometry (Figure 1a). Before characterization, graphene samples were washed and annealed in a vacuum chamber to remove polymer residuals and water. Ellipsometry data were analyzed and fitted without any additional assumptions such as the presence of water or any other medium on or under the graphene. To ensure the accuracy of our ellipsometric analysis, we utilized optical transmission spectroscopy measurements and X-ray photoelectron spectroscopy (XPS) to analyze the elemental composition and contribution of residues from the transfer process on the graphene samples. All graphene samples were synthesized by the same method and the same manufacturer, so any discrepancies in optical response should not be related to graphene quality differences. For additional analysis, we performed Raman spectroscopy, atomic-force, and scanning electron microscopy to evaluate the quality, thickness, and uniformity of CVD graphene on different substrates. 
a

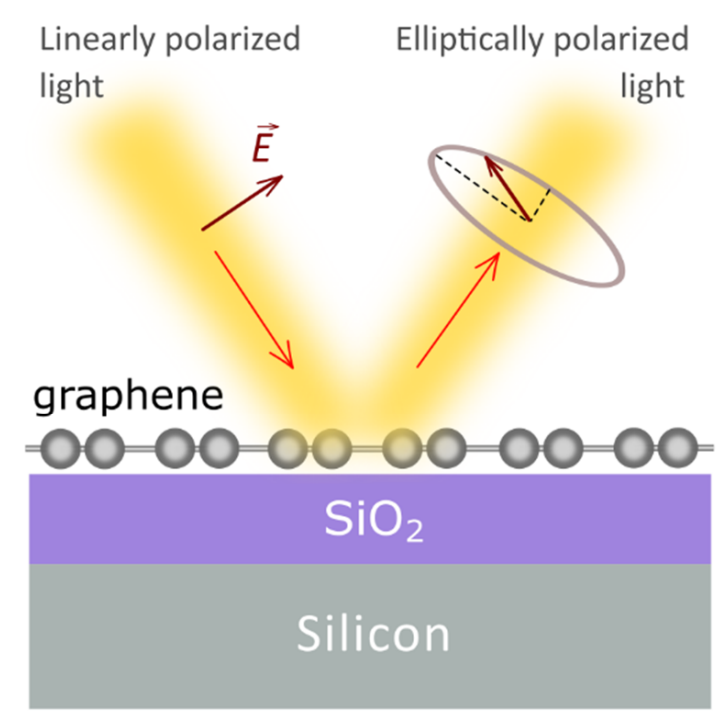

e



b

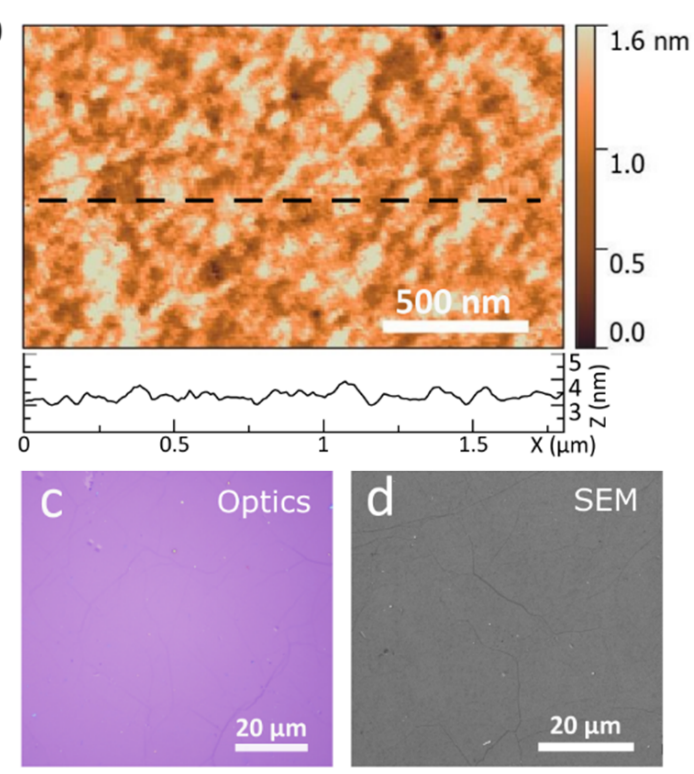

f

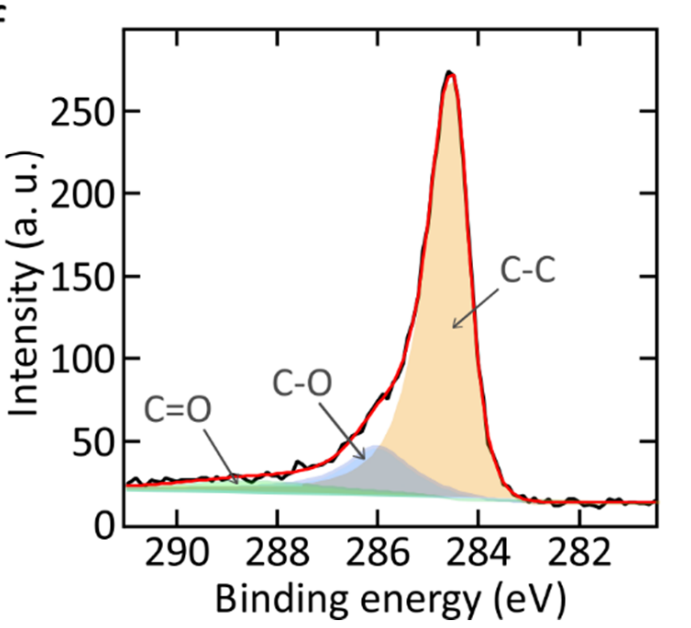

Figure 1. (a) The scheme of the ellipsometry measurements. (b) AFM topography mapping of single-layer graphene on $\mathrm{SiO}_{2} / \mathrm{Si}$ substrate with line profile across the surface. The scan area was $1.8 \times 1.1 \mu \mathrm{m}^{2}$. (c) Optical image of the graphene on top of $\mathrm{SiO}_{2} / \mathrm{Si}$ substrate. (d) SEM image of the graphene reveals a high crystallinity of the samples with the crystallite size larger than $10 \mu \mathrm{m}$. (e) Raman spectrum of graphene transferred on $\mathrm{SiO}_{2} / \mathrm{Si}$ substrate, at excitation wavelength $\lambda=532 \mathrm{~nm}$. (f) Results of XPS fitting analysis of the C1s core level signal obtained for the monolayer graphene on $\mathrm{SiO}_{2} / \mathrm{Si}$.

\section{Results and Discussion}

\subsection{Sample Preparation and Characterization}

All monolayer graphene films were prepared (see Methods) through chemical vapor deposition. Monolayers of graphene grown initially on copper foil were wet-transferred to three various substrates: $\mathrm{SiO}_{2}(285 \mathrm{~nm}) / \mathrm{Si}$, fused quartz, and optical glass. Before measurements, graphene samples were washed and annealed in a vacuum chamber to remove polymer residuals and water. The high quality of monolayer graphene films was confirmed by atomic force microscopy (AFM), optical microscopy, and scanning electron microscopy (SEM). The AFM scan image of the graphene on $\mathrm{SiO}_{2} / \mathrm{Si}$ in Figure 1 b confirms a small surface roughness typical for monolayer graphene surfaces. Large-scale optical microscopy images in Figures 1c and A1a,b, and SEM image of the graphene surface in Figure $1 \mathrm{~d}$ demonstrate that graphene uniformly covers more than $97 \%$ of the substrate surface without voids showing good crystallinity of the graphene sample with the crystallite size $>10 \mu \mathrm{m}$. To characterize the thickness and quality of monolayer graphene on different substrates, we performed Raman spectroscopy measurements (Figures 1e and A2a,b) [36,37]. 
The ratio of 2D $\left(2689 \mathrm{~cm}^{-1}\right)$ and $\mathrm{G}\left(1587 \mathrm{~cm}^{-1}\right)$ bands (>2) indicates the single layer of graphene, and the low intensity of the $\mathrm{D}$ band $\left(1347 \mathrm{~cm}^{-1}\right)$, shows its high quality (Figure 1e). Additionally, the 2D band exhibits a sharp Lorentzian peak, specific to a single layer of graphene [38].

Next, to analyze the presence of residues (from the transfer process) and their contribution to the accuracy of graphene dielectric response characterization, we performed X-ray photoelectron spectroscopy. Figure $1 \mathrm{f}$ shows the analysis of the $\mathrm{C} 1 \mathrm{~s}$ core level constituents for the graphene on $\mathrm{SiO}_{2} / \mathrm{Si}$ sample, in which the percentage contribution of sp2-graphene, $\mathrm{C}-\mathrm{O}$ and $\mathrm{C}=\mathrm{O}$ bonds to $\mathrm{C} 1 \mathrm{~s}$ are $80.72 \%, 14.89 \%$ and $4.39 \%$, respectively. To investigate the surface composition on top and under the graphene, we made the angle-resolved XPS measurements for the C1s and O1s core level signals in the range of take-off angles from $10^{\circ}$ to $70^{\circ}$ (measured from the sample surface) that can change the explored depth (see Figure A3) [28]. Both XPS analyses of the C1s and O1s core level signals in Figure A3 demonstrates a small contribution of $\mathrm{C}-\mathrm{O}$ and $\mathrm{C}=\mathrm{O}$ bonds, suggesting the negligible residua of polymer on the graphene surface. As the angle decreases, the relative intensity of the $\mathrm{C}-\mathrm{O}, \mathrm{C}=\mathrm{O}$ lines in the decomposition of the $\mathrm{C} 1$ s spectra and $\mathrm{O}-\mathrm{C}$ lines in the $\mathrm{O} 1 \mathrm{~s}$ spectra increases. Consequently, these lines are associated with surface states corresponding to adsorbed molecules and polymethylmethacrylate (PMMA) residues. Oxygen anions in $\mathrm{SiO}_{2}$ and $\mathrm{H}_{2} \mathrm{O}$ share the same binding energy region, and their contributions are indistinguishable. However, from the fact that the relative intensity of the $\mathrm{SiO}_{2}+\mathrm{H}_{2} \mathrm{O}$ lines in the decomposition of the O1s spectra decreases monotonically with decreasing angle, it can be concluded that $\mathrm{H}_{2} \mathrm{O}$ does not make a significant contribution to the total intensity of these lines. Indeed, as the angle decreases, photoelectrons are detected from a smaller sample depth. Therefore, the contribution from $\mathrm{SiO}_{2}$ should decrease. If the water concentration in the sample is significant, it partially compensates for the reduced contribution from $\mathrm{SiO}_{2}$. As a result, a nonmonotonic change in the intensity of the $\mathrm{SiO}_{2}+$ $\mathrm{H}_{2} \mathrm{O}$ line is observed [28]. Noteworthy, the evolution of constituents of the XPS spectra (Figure A3) with the increasing explored depth did not reveal the presence of contamination interlayers (water, PMMA) [28]. Thus they are not considered in the determination of graphene optical constants.

\subsection{Dielectric Response Analysis}

We performed spectroscopic ellipsometry (SE) measurements at multiple incident angles to explore the dielectric response of graphene samples, Figure 2a-c. SE measures the change in light polarization upon reflection from a sample in terms of $\Psi$ and $\Delta$ defined through equation $\tan (\Psi) \exp (\mathrm{i} \Delta)=r_{\mathrm{p}} / r_{\mathrm{s}}$, where $r_{\mathrm{p}}$ and $r_{\mathrm{s}}$ are sample's reflection amplitude for $\mathrm{p}$ - and s-polarized light. First, we analyzed these $\Psi$ and $\Delta$ data by point-by-point inversion to get the initial results presented in Figure $2 \mathrm{~d}$. In this approach, optical constants of graphene are varied independently for each wavelength until the best match with the experiment is achieved, allowing to get initial values for the dielectric response of graphene. Then, the dielectric function is fitted by the Drude-Lorentz oscillator model (see Methods and Table 1), which takes into account the optical response of quasi-free electrons (Drude oscillator) and graphene van Hove singularity at $\pi$-to- $\pi^{*}$ interband transition (Lorentz oscillator).Such a fitting approach yields smooth and Kramers-Kronig-consistent dielectric functions compared to noisy point-by-point results (Figure 2d). We calculated the transmittance spectrum based on retrieved optical constants, which is in good agreement with the experimental one as illustrated in Figure 2e, thereby validating the acquired dielectric function. 

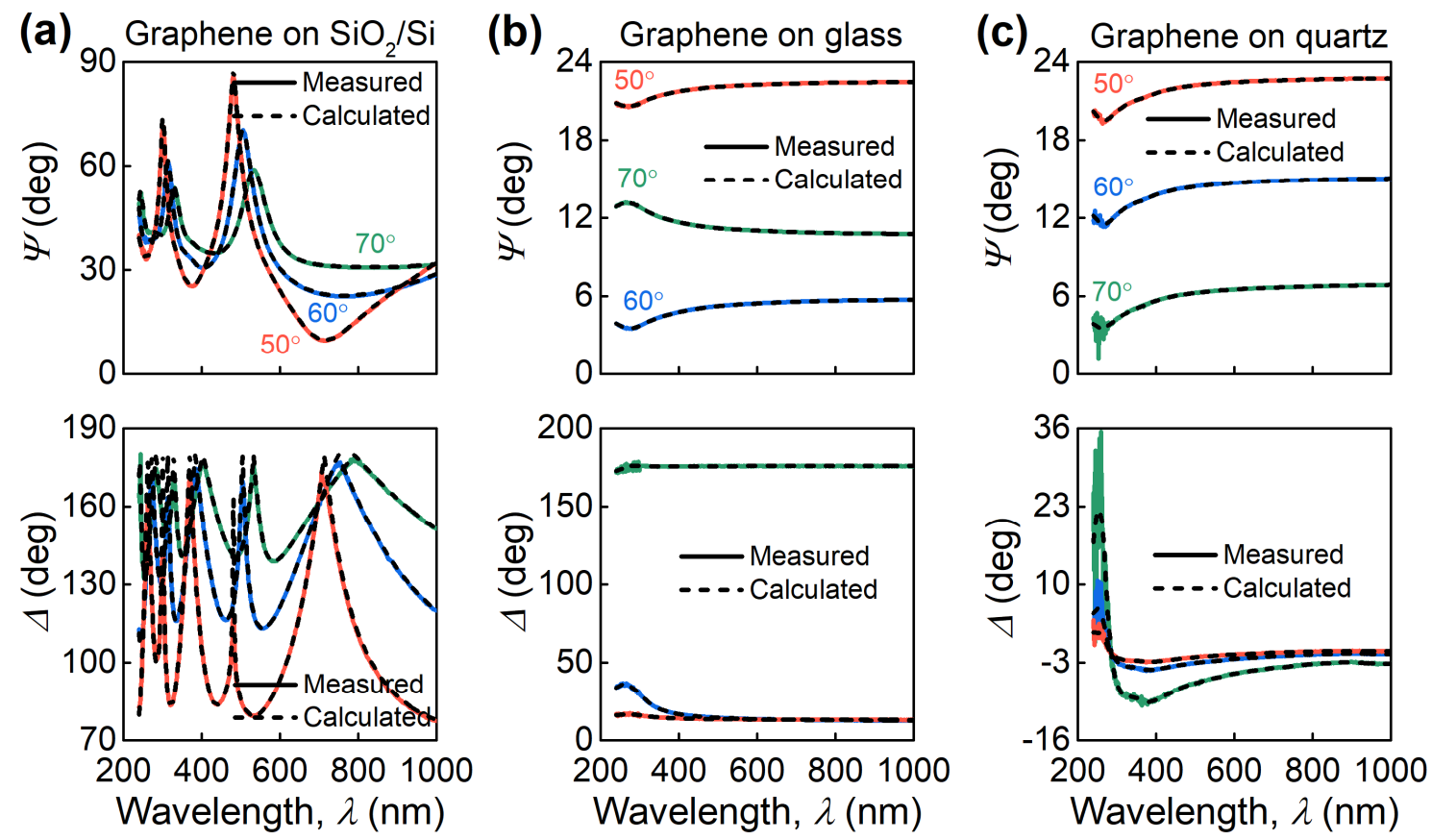

(d)

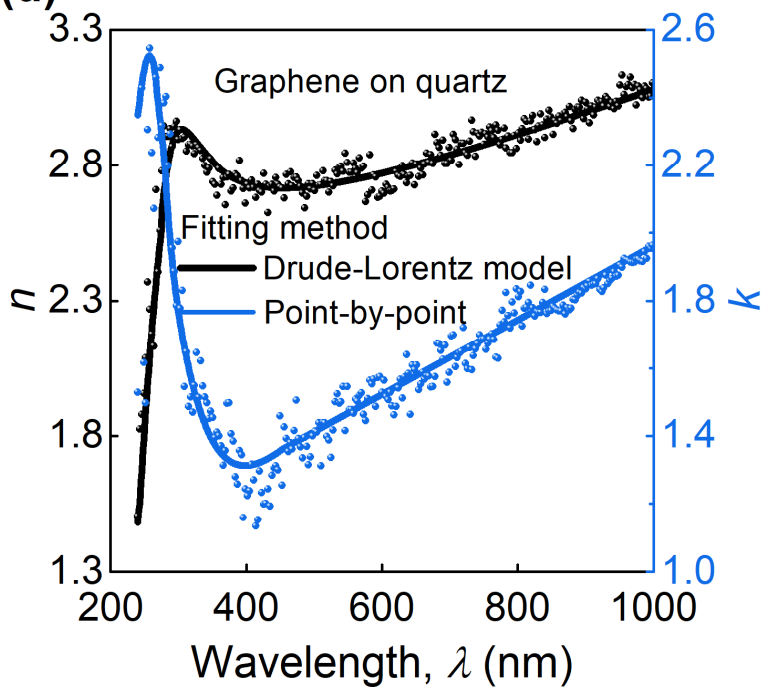

(e)

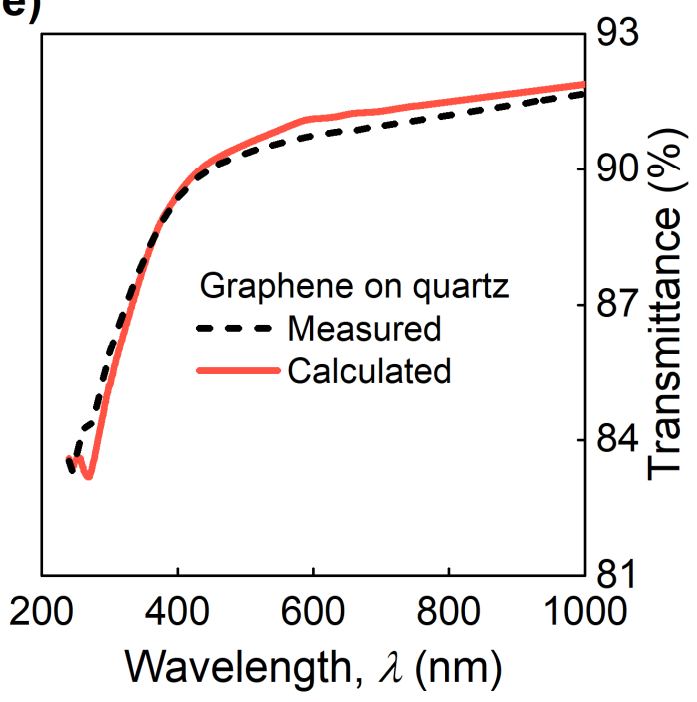

Figure 2. Plots of the measured (experiment) and calculated (model) ellipsometric spectra ( $\Psi$ and $\Delta$ ) of monolayer graphene on different substrates: (a) $\mathrm{SiO}_{2} / \mathrm{Si}$, (b) glass, and (c) quartz. (d) Refractive index $n$ and extinction coefficient $k$ of monolayer graphene on quartz acquired from SE analysis using different models. (e) Measured (red line) and calculated (black dashed line) transmittance spectra match perfectly within spectrophotometer accuracy (1\%).

Table 1. Drude-Lorentz oscillators parameters for graphene on $\mathrm{SiO}_{2} / \mathrm{Si}$, quartz, and glass.

\begin{tabular}{cccccc}
\hline Substrate & $A_{\mathbf{L}}$ & $\boldsymbol{B}_{\mathbf{L}}(\mathbf{e V})$ & $\boldsymbol{E}_{\mathbf{L}}(\mathbf{e V})$ & $\boldsymbol{\rho}\left(\mathbf{1 0}^{-\mathbf{4}} \mathbf{\Omega} \cdot \mathbf{m}\right)$ & $\boldsymbol{\tau}(\mathbf{f s})$ \\
\hline $\mathrm{SiO}_{2} / \mathrm{Si}$ & 8.0531 & 1.5891 & 4.5715 & 5.3668 & 0.60878 \\
Quartz & 8.4404 & 1.6275 & 4.6179 & 4.8038 & 0.65817 \\
Glass & 7.8742 & 1.5650 & 4.5201 & 5.2734 & 0.61750 \\
\hline
\end{tabular}

Interestingly, graphene's optical response is similar in shape to other carbon materials such as graphite [39] and single-walled carbon nanotube (SWCNT) films [40], as illustrated in Figure 3. More importantly, the optical constants of graphene are almost identical to graphite. Consequently, in the considered wavelength range (240-1000 nm), graphene's optical response is also similar to any number of graphene layers. As a result, the obtained 
optical constants of graphene could also be used for bilayer, trilayer, and further graphene layers up to graphite. This observation is in total agreement with the results of previous studies [41].
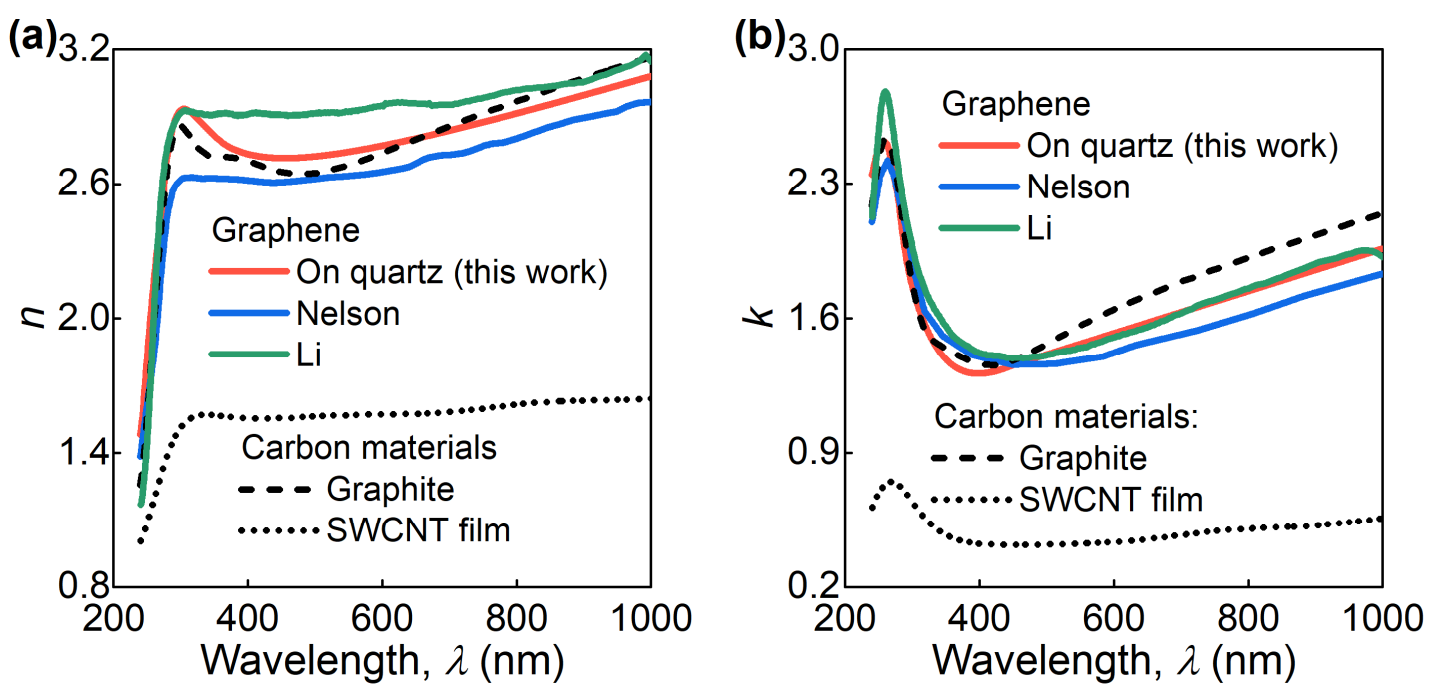

Figure 3. Comparison of the measured (red lines) refractive index $n$ (a) and extinction coefficient $k$ (b) and some previous results for CVD graphene on glass (blue lines) and quartz (green lines) substrates obtained without any assumptions in the ellipsometric model and carbon materials such as graphite (dashed lines) and SWCNT film (dotted lines). The data of Nelson, Li, Graphite, and SWCNT film are adopted from refs. [26,29,39,40], respectively.

We also investigated graphene transferred on different substrates (quartz, glass, and $\mathrm{SiO}_{2} / \mathrm{Si}$ ) to explore substrate effect, which could explain discrepancies observed in the literature for graphene optical constants. We proceeded with the same procedure as for quartz: first, with the point-by-point approach and the Drude-Lorentz model afterward. The resulting parameters for the Drude-Lorentz model and corresponding optical constants for graphene on quartz, glass, and $\mathrm{SiO}_{2} / \mathrm{Si}$ substrates are collected in Table 1 and Figure $4 \mathrm{a}$. Clearly, the dielectric function is reproduced with $95 \%$ accuracy. Therefore, the substrate effect is negligible for graphene optical constants.


Figure 4. (a) The measured refractive index $n$ and extinction coefficient $k$ of CVD graphene on three different substrates. For the tabular data, see Appendix A Table A1. (b) Comparison of the measured optical constants (red lines) and some previous results for CVD graphene obtained under the assumption of the presence of nanometer-thick layers of water in the ellipsometric model (cyan and magenta lines). Solid, $n$, and dashed, $k$, lines. The data of Ochoa-Martinez and Castriota are adopted from refs. [28,31], respectively. 
In contrast, the inclusion of a nanometer-thick water layer in the optical model to consider graphene contamination noticeably changes the result as seen from Figure $4 \mathrm{~b}$ (there is a large discrepancy by more than $20 \%$ ). Although this approach allows the inclusion of graphene nonidealities, current devices utilize encapsulated graphene where contamination is minimal. Thus, graphene should be analyzed immediately after the cleaning procedure, as in our work, to enable predictive capabilities for photonic applications.

\subsection{Applications}

To illustrate the importance of refining the optical constants of graphene, we consider two examples of the devices, including graphene-based heterostructures. One graphene layer could not significantly affect some systems' optical response because it is too thin, but when several layers of graphene are included in a metamaterial [42,43], the effect of the dielectric function's inaccuracy becomes apparent. Firstly, we evaluate the optical response of a metamaterial consisting of alternating layers of graphene and h-BN [44], placed on the surface of a standard surface plasmon resonance (SPR) chip, a $\mathrm{SiO}_{2}$ prism with a 40-nm-thick gold film [45]. Such metamaterial enhances the SPR chip's performance due to the rise of optical sensitivity (Figure $5 b$ ) and graphene's ability to effectively adsorb the studied molecules [12,13]. Figure 5a shows the calculated reflectance of the chip with the optimal (see Figure 5b) 10-nm-thick layer of the metamaterial at a free-space wavelength of $800 \mathrm{~nm}$. Evidently, both the resonance depth and position are strongly dependent on the optical properties of graphene. The expected sensitivity of the considered sensor, shown in Figure 5 b, varies by more than $10 \%$ when different sources of graphene's optical properties are used in calculations.
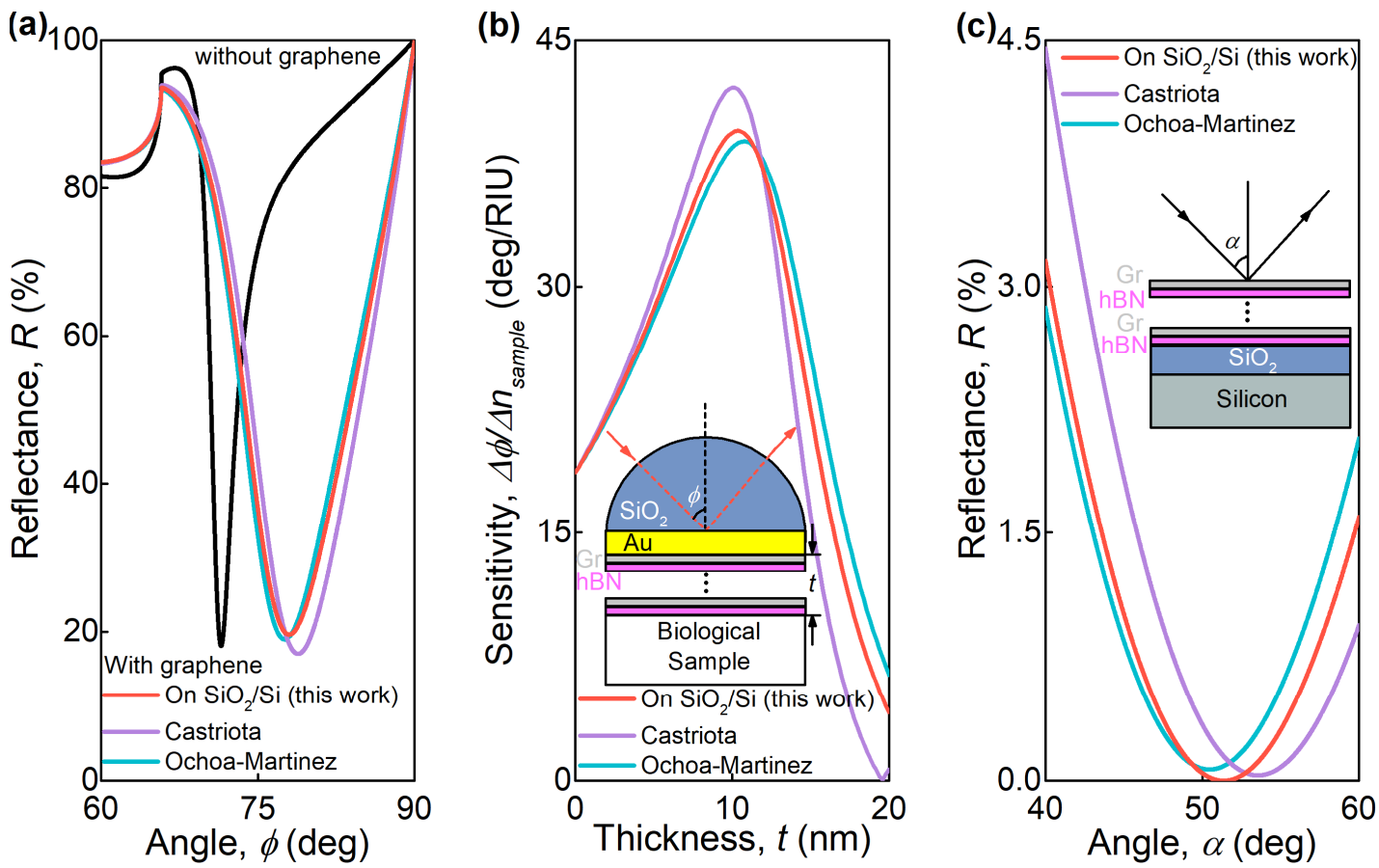

Figure 5. The operation of the SPR sensor based on $\mathrm{SiO}_{2} / \mathrm{Au}$ (Au-thickness equals to $40 \mathrm{~nm}$ ) chip with the metamaterial, consisting of alternating layers graphene and h-BN $(\mathbf{a}, \mathbf{b})$. (a) The dependence of the reflection coefficient as a function of the incident angle at the $800 \mathrm{~nm}$ wavelength and 10-nm-thick metamaterial. The black curve corresponds to the absence of the metamaterial layer; red, blue, and green curves calculated using different optical properties of graphene, that were reported. (b) The dependence of the sensitivity of this SPR sensor on the thickness of the metamaterial. (c) The TM-mode reflectance from the 20-nm-thick metamaterial, consisting of alternating layers graphene and h-BN, placed on the $\mathrm{SiO}_{2} / \mathrm{Si} \mathrm{substrate}$ $\left(\mathrm{SiO}_{2}\right.$ thickness equals to $\left.280 \mathrm{~nm}\right)$, as a function of the incident angle at $530 \mathrm{~nm}$ free-space wavelength. Red, purple and blue-green curves were calculated using optical properties of graphene from different sources. The optical constants of graphene of Ochoa-Martinez and Castriota are adopted for calculations from refs. [28,31], respectively. 
In addition, we consider the same metamaterial $(\mathrm{Gr} / \mathrm{h}-\mathrm{BN})$ with a $20 \mathrm{~nm}$ thickness on top of the standard $\mathrm{SiO}_{2} / \mathrm{Si}$ substrate to investigate its zero reflection conditions giving rise to phase singularity used in meta-optics design [46]. We evaluate the incident-angledependent reflectance of TM-wave at a wavelength of $530 \mathrm{~nm}$ using graphene's optical properties reported by different sources. For optical constants obtained in our work, this structure exhibits zero reflection at an incident angle of approximately 51 degrees, while in the case of the optical constants obtained in [31], the zero reflection angle shifts to 53 degrees. In contrast, for the optical constants obtained in [28], zero reflection is not achieved.

\section{Materials and Methods}

\subsection{Materials}

Full area coverage graphene samples were purchased from Graphene Laboratories Inc., Ronkonkoma, NY, USA (https:/ / graphene-supermarket.com accessed on 27 April 2021). Graphene was grown via chemical vapor deposition on a copper foil and wettransferred onto silicon wafer covered by a 285-nm-thick layer of $\mathrm{SiO}_{2}$, glass (Corning EAGLE XG, New York, NY, USA), and fused quartz (Volume Precision Glass, Inc. G.E. 124, Santa Rosa, CA, USA) substrates. Monolayer graphene covered about $95 \%$ of the substrate area. Before measurements, graphene substrates were washed in acetone and isopropyl alcohol baths and annealed at $200^{\circ} \mathrm{C}$ in a vacuum chamber $10^{-6}$ Torr for more than $1 \mathrm{~h}$ to remove residual PMMA and water after the transfer process.

\subsection{Raman Characterization}

The experimental setup used for Raman measurements was a confocal scanning Raman microscope Horiba LabRAM HR Evolution (HORIBA Ltd., Kyoto, Japan). All measurements were carried out using linearly polarized excitation at wavelength $532 \mathrm{~nm}$, 1800 lines/mm diffraction grating, and $\times 100$ objective (N.A. $=0.90)$. Meanwhile, we used unpolarized detection to have a significant signal-to-noise ratio. The spot size was $\sim 0.4 \mu \mathrm{m}$. The Raman spectra were recorded with $0.9 \mathrm{~mW}$ incident powers and an integration time of $10 \mathrm{~s}$ at each point. The statistics were collected at least 10 points for each sample.

\subsection{XPS Characterization}

For the detailed study of the transferred CVD graphene optical response, we carried out the angle-dependent measurements of the O1s and C1s core level XPS spectra to determine contaminants' presence on top of graphene. Three take-off angles, $10^{\circ}, 40^{\circ}$ and $70^{\circ}$ (measured from the surface of the sample), were used to change the explored depth in XPS measurements. In order to investigate the contributions of different bonds, the core-level spectra of the O1s and C1s were decomposed using the XPS peak fitting software package.

\subsection{Atomic Force Microscopy, Optical Visualization, Scanning Electron Microscopy}

The roughness and homogeneity of the graphene were measured by an atomic force microscope (NT-MDT Ntegra, Moscow, Russia). The surface images ( $2400 \times 2400$ pixels) of the graphene samples were captured by an optical microscope (Nikon LV150, Tokyo, Japan) with a digital camera DS-Fi3. To investigate graphene's surface, we additionally used the scanning electron microscope using the acceleration voltage of $3 \mathrm{kV}$ (JEOL JSM-7001F, Tokyo, Japan).

\subsection{Ellipsometry Characterization}

Spectroscopic ellipsometry was performed at room temperature at multiple incident angles $\left(50^{\circ}, 60^{\circ}, 70^{\circ}\right)$, over a broad spectral range from 240 to $1000 \mathrm{~nm}(1.24-5.17 \mathrm{eV})$. The measurements were done using a variable-angle spectroscopic ellipsometer (VASE, J.A. Woollam Co., Lincoln, NE, USA). To avoid any crushes or large tears of graphene, we measured samples in different areas, excluding from consideration the areas close to 
the edges where graphene's substrate coverage is minimal. For ellipsometry analysis we implemented the Drude-Lorentz model [47]:

$$
\begin{aligned}
& \varepsilon=\varepsilon_{\text {Drude }}+\varepsilon_{\text {Lorentz }}= \\
& =1-\frac{\hbar^{2}}{\varepsilon_{0} \rho\left(\tau E^{2}+i \hbar E\right)}+\frac{A_{\mathrm{L}} B_{\mathrm{L}} E_{\mathrm{L}}}{E_{\mathrm{L}}^{2}-E^{2}-i B_{\mathrm{L}} E^{\prime}}
\end{aligned}
$$

where $\varepsilon_{0}$ is the vacuum dielectric constant, $\hbar$ is Planck's constant, $\rho$ is the resistivity, $\tau$ is the mean scattering time, and $A_{\mathrm{L}}, B_{\mathrm{L}}$, and $E_{\mathrm{L}}$ are the amplitude, the linewidth, and the resonance energy of the Lorentz oscillator.

\section{Conclusions}

To summarize, we have presented a UV-visible-near-IR $(240-1000 \mathrm{~nm})$ spectroscopic ellipsometry of graphene grown by chemical vapor deposition and transferred onto glass, quartz, and $\mathrm{SiO}_{2} / \mathrm{Si}$ substrates. To obtain an accurate dielectric function suitable for engineering purposes, we thoroughly cleaned and annealed our samples prior to measurements and carried out XPS analysis confirming an ultra-low level of contamination on the graphene's surface. As a result, we achieved good convergence using a simple ellipsometric model comprising layers of substrate and graphene layer, without auxiliary layers, which are typically introduced to account for residual water and PMMA. The accuracy of the measured optical response was further confirmed by transmittance measurements, which demonstrate remarkable agreement with calculations based on the measured optical constants. Notably, our optical constants show less than $5 \%$ variation with changes of the substrate and are very similar to those of graphite, which implies that the measured optical constants are applicable to multilayer graphene structures as well. From a broader perspective, our work provides accurate and universal graphene optical response for the design of photonic devices.

Author Contributions: M.A.E.-S., G.A.E., K.V.V., G.I.T., S.M.N., A.A.V. (Andrey A. Vyshnevyy), A.V.A. and V.S.V. proposed the concept, conceived and designed the experiments and wrote original draft. M.A.E.-S., G.A.E., R.I.R., G.I.T., D.I.Y., N.V.D., A.B.N., V.R.S., A.A.V. (Artem A. Voronov), S.M.N and A.M.M. performed the measurements and analyzed the data. K.V.V. and A.A.V. (Andrey A. Vyshnevyy) provided theoretical support. All authors discussed the results and contributed to manuscript preparation. M.A.E.-S. and G.A.E. contributed equally to this work and should be considered co-first authors. All authors have read and agreed to the published version of the manuscript.

Funding: The experimental work was supported by the Russian Science Foundation, grant No. 19-79-00350. The numerical analysis of multilayer structures was supported by the Russian Science Foundation, grant No. 20-79-00349.

Institutional Review Board Statement: Not applicable.

Informed Consent Statement: Not applicable.

Data Availability Statement: The data presented in this study are available on request from the corresponding author.

Acknowledgments: The authors thank the MIPT's Shared Research Facilities Center for the use of their equipment.

Conflicts of Interest: The authors declare no conflict of interest. 


\section{Appendix A}

a

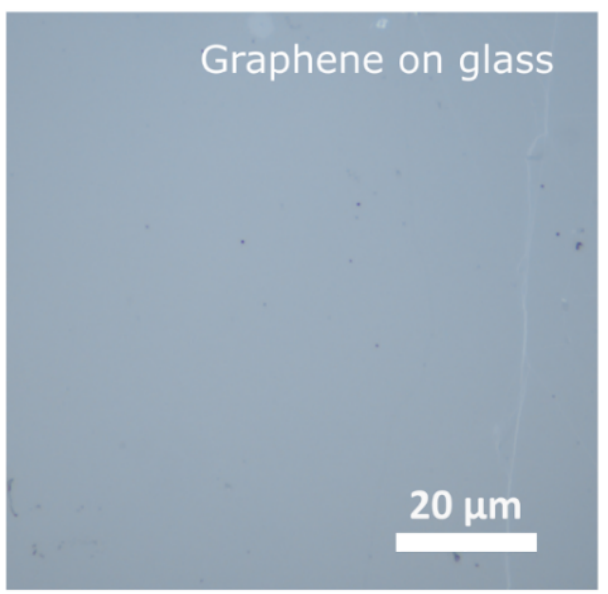

b

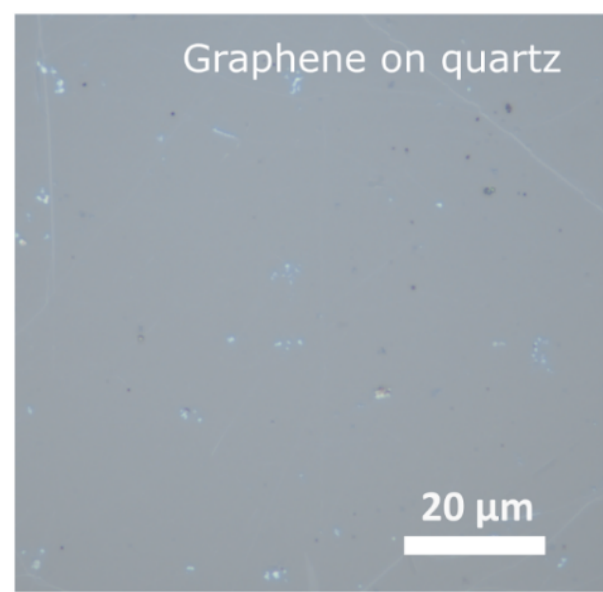

Figure A1. Optical images of the graphene on top of the glass (a) and quartz (b) substrates.
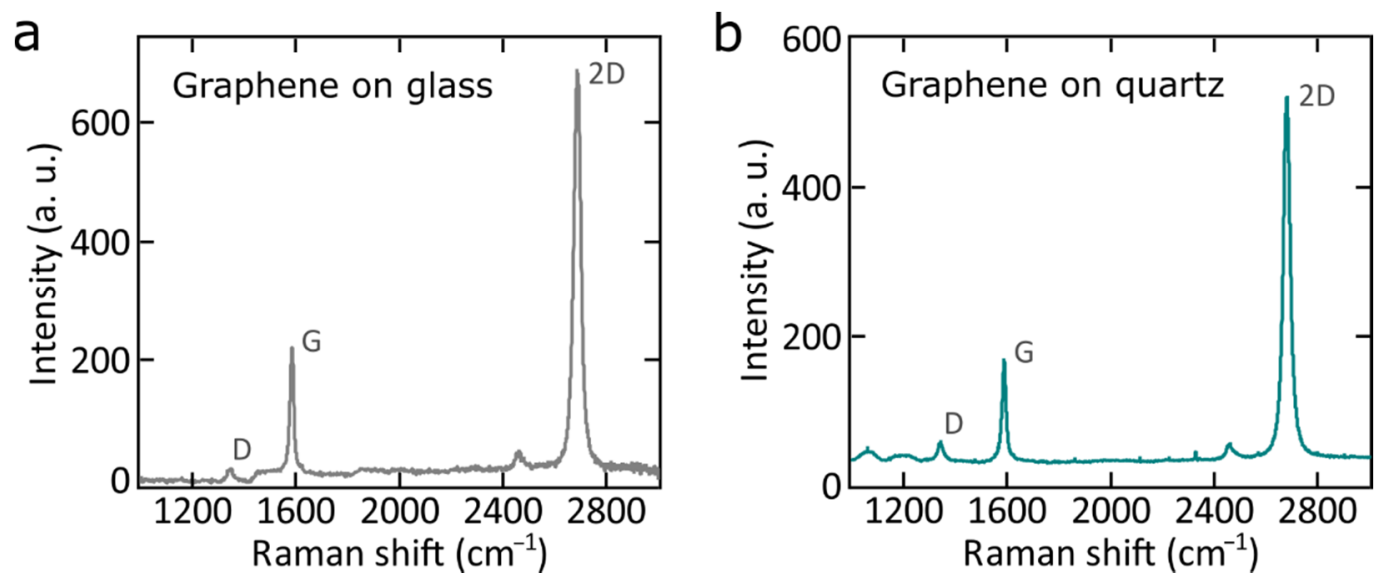

Figure A2. Raman spectra of graphene transferred on glass (a) and quartz (b) substrates, obtained with an excitation wavelength of $\lambda=532 \mathrm{~nm}$.
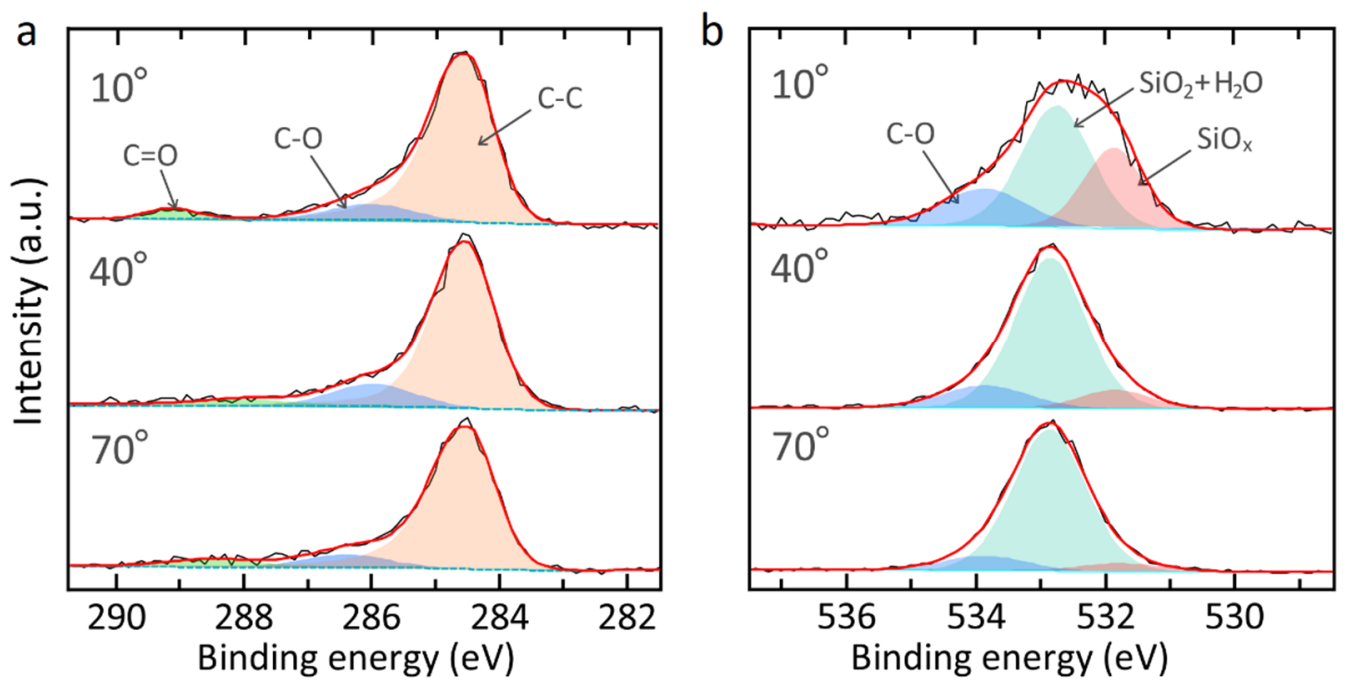

Figure A3. Results of the angle-resolved XPS measurements of the graphene transferred onto $\mathrm{SiO}_{2} / \mathrm{Si}$ substrate. Decomposition of $\mathrm{C} 1 \mathrm{~s}$ (a) and $\mathrm{O} 1 \mathrm{~s}$ (b) core level signals into their constituents. Take-off angle (measured from the sample surface) increases from $10^{\circ}$ to $70^{\circ}$; correspondingly, the explored depth of the sample is increased. 
Table A1. Tabulated optical constants for graphene on different substrates.

\begin{tabular}{|c|c|c|c|c|c|c|}
\hline \multirow{3}{*}{$\lambda(\mathrm{nm})$} & \multicolumn{6}{|c|}{ Substrate } \\
\hline & \multicolumn{2}{|c|}{$\mathrm{SiO}_{2} / \mathrm{Si}$} & \multicolumn{2}{|c|}{ Quartz } & \multicolumn{2}{|c|}{ Glass } \\
\hline & $n$ & $k$ & $n$ & $k$ & $n$ & $k$ \\
\hline 240 & 1.5018 & 2.3036 & 1.4913 & 2.3303 & 1.5033 & 2.2261 \\
\hline 260 & 2.1096 & 2.4266 & 2.1676 & 2.5087 & 2.0678 & 2.3145 \\
\hline 280 & 2.6262 & 2.1543 & 2.7044 & 2.1765 & 2.5344 & 2.1402 \\
\hline 300 & 2.866 & 1.7567 & 2.9296 & 1.7667 & 2.7947 & 1.8313 \\
\hline 320 & 2.8629 & 1.496 & 2.9093 & 1.5577 & 2.8391 & 1.5565 \\
\hline 340 & 2.8004 & 1.3584 & 2.8452 & 1.4296 & 2.7962 & 1.389 \\
\hline 360 & 2.7408 & 1.2902 & 2.7901 & 1.3557 & 2.7397 & 1.3133 \\
\hline 380 & 2.6956 & 1.2595 & 2.7539 & 1.3209 & 2.6909 & 1.2874 \\
\hline 400 & 2.6637 & 1.2498 & 2.7338 & 1.3126 & 2.6531 & 1.2834 \\
\hline 420 & 2.6426 & 1.2529 & 2.7222 & 1.3219 & 2.6255 & 1.2907 \\
\hline 440 & 2.6298 & 1.2639 & 2.7167 & 1.343 & 2.6063 & 1.3044 \\
\hline 460 & 2.6234 & 1.28 & 2.7158 & 1.3683 & 2.5938 & 1.3216 \\
\hline 480 & 2.6221 & 1.2997 & 2.7182 & 1.3905 & 2.5864 & 1.3408 \\
\hline 500 & 2.6247 & 1.3216 & 2.7232 & 1.4125 & 2.5838 & 1.3609 \\
\hline 520 & 2.6305 & 1.3451 & 2.7301 & 1.4345 & 2.5849 & 1.3815 \\
\hline 540 & 2.6389 & 1.3697 & 2.7386 & 1.4565 & 2.5888 & 1.4022 \\
\hline 560 & 2.6493 & 1.395 & 2.7483 & 1.4785 & 2.5952 & 1.4228 \\
\hline 580 & 2.6614 & 1.4207 & 2.7591 & 1.5006 & 2.6044 & 1.4433 \\
\hline 600 & 2.6749 & 1.4465 & 2.7708 & 1.5226 & 2.6167 & 1.4635 \\
\hline 620 & 2.6894 & 1.4725 & 2.7832 & 1.5446 & 2.6316 & 1.4836 \\
\hline 640 & 2.7049 & 1.4984 & 2.7962 & 1.5666 & 2.6485 & 1.5035 \\
\hline 660 & 2.721 & 1.5243 & 2.8098 & 1.5886 & 2.6663 & 1.5233 \\
\hline 680 & 2.7376 & 1.55 & 2.8238 & 1.6107 & 2.6843 & 1.5429 \\
\hline 700 & 2.7546 & 1.5756 & 2.8382 & 1.6327 & 2.7021 & 1.5624 \\
\hline 720 & 2.7719 & 1.5976 & 2.8529 & 1.6547 & 2.7196 & 1.5819 \\
\hline 740 & 2.7893 & 1.6196 & 2.868 & 1.6767 & 2.7372 & 1.6013 \\
\hline 760 & 2.8067 & 1.6416 & 2.8834 & 1.6988 & 2.7547 & 1.6206 \\
\hline 780 & 2.824 & 1.6637 & 2.899 & 1.7208 & 2.7723 & 1.64 \\
\hline 800 & 2.8411 & 1.6857 & 2.9148 & 1.7428 & 2.7898 & 1.6593 \\
\hline 820 & 2.8576 & 1.7077 & 2.9307 & 1.7648 & 2.8074 & 1.6786 \\
\hline 840 & 2.8754 & 1.7297 & 2.9469 & 1.7868 & 2.825 & 1.698 \\
\hline 860 & 2.8933 & 1.7518 & 2.9632 & 1.8089 & 2.8425 & 1.7174 \\
\hline 880 & 2.9115 & 1.7738 & 2.9796 & 1.8309 & 2.8601 & 1.7368 \\
\hline 900 & 2.9298 & 1.7958 & 2.9962 & 1.8529 & 2.8776 & 1.7563 \\
\hline 920 & 2.9483 & 1.8178 & 3.0128 & 1.8749 & 2.8952 & 1.7758 \\
\hline 940 & 2.9669 & 1.8398 & 3.0296 & 1.897 & 2.9128 & 1.7953 \\
\hline 960 & 2.9856 & 1.8619 & 3.0464 & 1.919 & 2.9303 & 1.8151 \\
\hline 980 & 3.0044 & 1.8839 & 3.0633 & 1.9412 & 2.9479 & 1.8356 \\
\hline 1000 & 3.0233 & 1.9059 & 3.0803 & 1.9623 & 2.9655 & 1.8571 \\
\hline
\end{tabular}

\section{References}

1. Bonaccorso, F.; Sun, Z.; Hasan, T.; Ferrari, A.C. Graphene photonics and optoelectronics. Nat. Photonics 2010, 4, 611-622. [CrossRef]

2. Bao, Q.; Loh, K.P. Graphene photonics, plasmonics, and broadband optoelectronic devices. ACS Nano 2012, 6, 3677-3694. [CrossRef]

3. Vakil, A.; Engheta, N. Transformation optics using graphene. Science 2011, 332, 1291-1294. [CrossRef] [PubMed]

4. Ferrari, A.C.; Bonaccorso, F.; Fal'Ko, V.; Novoselov, K.S.; Roche, S.; Bøggild, P.; Borini, S.; Koppens, F.H.L.; Palermo, V.; Pugno, N.; et al. Science and technology roadmap for graphene, related two-dimensional crystals, and hybrid systems. Nanoscale 2015, 7, 4598-4810. [CrossRef]

5. Grigorenko, A.N.; Polini, M.; Novoselov, K.S. Graphene plasmonics. Nat. Photonics 2012, 6, 749-758. [CrossRef]

6. Luongo, G.; Di Bartolomeo, A.; Giubileo, F.; Chavarin, C.A.; Wenger, C. Electronic properties of graphene/p-silicon Schottky junction. J. Phys. D Appl. Phys. 2018, 51, 255305. [CrossRef]

7. Luongo, G.; Grillo, A.; Giubileo, F.; Iemmo, L.; Lukosius, M.; Alvarado Chavarin, C.; Wenger, C.; Di Bartolomeo, A. Graphene Schottky junction on pillar patterned silicon substrate. Nanomaterials 2018, 9, 659. [CrossRef] [PubMed] 
8. Liu, M.; Yin, X.; Ulin-Avila, E.; Geng, B.; Zentgraf, T.; Ju, L.; Wang, F.; Zhang, X. A graphene-based broadband optical mod-ulator. Nature 2011, 474, 64-67. [CrossRef] [PubMed]

9. Sun, Z.; Martinez, A.; Wang, F. Optical modulators with 2D layered materials. Nat. Photonics 2016, 10, 227-238. [CrossRef]

10. Dalir, H.; Xia, Y.; Wang, Y.; Zhang, X. Athermal broadband graphene optical modulator with 35 GHz speed. ACS Photonics 2016, 3, 1564-1568. [CrossRef]

11. Gan, X.; Shiue, R.-J.; Gao, Y.; Meric, I.; Heinz, T.F.; Shepard, K.L.; Hone, J.; Assefa, S.; Englund, D. Chip-integrated ultrafast graphene photodetector with high responsivity. Nat. Photonics 2013, 7, 883-887. [CrossRef]

12. Wu, L.; Chu, H.S.; Koh, W.S.; Li, E.P. Highly sensitive graphene biosensors based on surface plasmon resonance. Opt. Express 2010, 18, 14395-14400. [CrossRef] [PubMed]

13. Stebunov, Y.V.; Aftenieva, O.A.; Arsenin, A.V.; Volkov, V.S. Highly sensitive and selective sensor chips with graphene-oxide linking layer. ACS Appl. Mater. Interfaces 2015, 7, 21727-21734. [CrossRef] [PubMed]

14. Li, X.; Zhu, H.; Wang, K.; Cao, A.; Wei, J.; Li, C.; Jia, Y.; Li, Z.; Li, X.; Wu, D. Graphene-on-silicon schottky junction solar cells. Adv. Mater. 2010, 22, 2743-2748. [CrossRef] [PubMed]

15. Lang, F.; Gluba, M.A.; Albrecht, S.; Rappich, J.; Korte, L.; Rech, B.; Nickel, N.H. Perovskite solar cells with large-area cvd-graphene for tandem solar cells. J. Phys. Chem. Lett. 2015, 6, 2745-2750. [CrossRef]

16. Ermolaev, G.A.; Stebunov, Y.V.; Vyshnevyy, A.A.; Tatarkin, D.E.; Yakubovsky, D.I.; Novikov, S.M.; Baranov, D.G.; Shegai, T.; Nikitin, A.Y.; Arsenin, A.V.; et al. Broadband optical properties of monolayer and bulk MoS 2 . NPJ 2D Mater. Appl. 2020, 4, 1-6. [CrossRef]

17. Ermolaev, G.A.; Yakubovsky, D.I.; Stebunov, Y.V.; Arsenin, A.V.; Volkov, V.S. Spectral ellipsometry of monolayer transition metal dichalcogenides: Analysis of excitonic peaks in dispersion. J. Vac. Sci. Technol. B 2020, 38, 014002. [CrossRef]

18. Kravets, V.G.; Grigorenko, A.N.; Nair, R.R.; Blake, P.; Anissimova, S.; Novoselov, K.S.; Geim, A.K. Spectroscopic ellipsometry of graphene and an exciton-shifted van Hove peak in absorption. Phys. Rev. B 2010, 81, 155413. [CrossRef]

19. Matković, A.; Ralević, U.; Chhikara, M.; JakovljeviĆ, M.M.; Jovanović, D.; Bratina, G.; Gajić, R. Influence of transfer residue on the optical properties of chemical vapor deposited graphene investigated through spectroscopic ellipsometry. J. Appl. Phys. 2013, 114, 093505. [CrossRef]

20. Wurstbauer, U.; Röling, C.; Wurstbauer, U.; Wegscheider, W.; Vaupel, M.; Thiesen, P.H.; Weiss, D. Imaging ellipsometry of graphene. Appl. Phys. Lett. 2010, 97, 231901. [CrossRef]

21. Matković, A.; Beltaos, A.; Milićević, M.; Ralević, U.; Vasić, B.; Jovanović, D.; Gajić, R. Spectroscopic imaging ellipsometry and Fano resonance modeling of graphene. J. Appl. Phys. 2012, 112, 123523. [CrossRef]

22. Weber, J.J.-W.; Calado, V.E.; Van De Sanden, M.R. Optical constants of graphene measured by spectroscopic ellipsometry. Appl. Phys. Lett. 2010, 97, 091904. [CrossRef]

23. Nelson, F.; Sandin, A.; Dougherty, D.B.; Aspnes, D.E.; Rowe, J.E.; Diebold, A.C. Optical and structural characterization of epitaxial graphene on vicinal 6H-SiC(0001)-Si by spectroscopic ellipsometry, Auger spectroscopy, and STM. J. Vac. Sci. Technol. B 2012, 30 , 04E106. [CrossRef]

24. Boosalis, A.; Hofmann, T.; Darakchieva, V.; Yakimova, R.; Schubert, M. Visible to vacuum ultraviolet dielectric functions of epitaxial graphene on $3 \mathrm{C}$ and $4 \mathrm{H} \mathrm{SiC}$ polytypes determined by spectroscopic ellipsometry. Appl. Phys. Lett. 2012, 101, 011912. [CrossRef]

25. Darakchieva, V.; Boosalis, A.; Zakharov, A.A.; Hofmann, T.; Schubert, M.; Tiwald, T.E.; Iakimov, T.; Vasiliauskas, R.; Ya-kimova, R. Large-area microfocal spectroscopic ellipsometry mapping of thickness and electronic properties of epitaxial graphene on Si- and C-face of 3C-SiC(111). Appl. Phys. Lett. 2013, 102, 213116. [CrossRef]

26. Nelson, F.J.; Kamineni, V.K.; Zhang, T.; Comfort, E.S.; Lee, J.U.; Diebold, A.C. Optical properties of large-area polycrystalline chemical vapor deposited graphene by spectroscopic ellipsometry. Appl. Phys. Lett. 2010, 97, 253110. [CrossRef]

27. Chang, Y.-C.; Liu, C.-H.; Liu, C.-H.; Zhong, Z.; Norris, T.B. Extracting the complex optical conductivity of mono- and bilayer graphene by ellipsometry. Appl. Phys. Lett. 2014, 104, 261909. [CrossRef]

28. Ochoa-Martínez, E.; Gabás, M.; Barrutia, L.; Pesquera, A.; Centeno, A.; Palanco, S.; Zurutuza, A.; Algora, C. Determination of a refractive index and an extinction coefficient of standard production of CVD-graphene. Nanoscale 2015, 7, 1491-1500. [CrossRef]

29. Li, W.; Cheng, G.; Liang, Y.; Tian, B.; Liang, X.; Peng, L.; Hight Walker, A.R.; Gundlach, D.J.; Nguyen, N.V. Broadband optical properties of graphene by spectroscopic ellipsometry. Carbon 2016, 99, 348-353. [CrossRef]

30. Song, B.; Gu, H.; Zhu, S.; Jiang, H.; Chen, X.; Zhang, C.; Liu, S. Broadband optical properties of graphene and HOPG investigated by spectroscopic Mueller matrix ellipsometry. Appl. Surf. Sci. 2018, 439, 1079-1087. [CrossRef]

31. Castriota, M.; Politano, G.G.; Vena, C.; De Santo, M.P.; Desiderio, G.; Davoli, M.; Cazzanelli, E.; Versace, C. Variable angle spectroscopic ellipsometry investigation of CVD-grown monolayer graphene. Appl. Surf. Sci. 2019, 467-468, 213-220. [CrossRef]

32. Matković, A.; Chhikara, M.; Milićević, M.; Ralević, U.; Vasić, B.; Jovanović, D.; Belic, M.R.; Bratina, G.; Gajic, R. Influence of a gold substrate on the optical properties of graphene. J. Appl. Phys. 2015, 117, 015305. [CrossRef]

33. Lin, Y.-C.; Lu, C.-C.; Yeh, C.-H.; Jin, C.; Suenaga, K.; Chiu, P.-W. Graphene annealing: How clean can it be? Nano Lett. 2012, 12, 414-419. [CrossRef]

34. Lupina, G.; Kitzmann, J.; Costina, I.; Lukosius, M.; Wenger, C.; Wolff, A.; Vaziri, S.; Östling, M.; Pasternak, I.; Krajewska, A.; et al. Residual Metallic contamination of transferred chemical vapor deposited graphene. ACS Nano 2015, 9, 4776-4785. [CrossRef] 
35. Yakubovsky, D.I.; Stebunov, Y.V.; Kirtaev, R.V.; Voronin, K.V.; Voronov, A.A.; Arsenin, A.V.; Volkov, V.S. Graphene-supported thin metal films for nanophotonics and optoelectronics. Nanomaterials 2018, 8, 1058. [CrossRef] [PubMed]

36. Ferrari, A.C.; Meyer, J.C.; Scardaci, V.; Casiraghi, C.; Lazzeri, M.; Mauri, F.; Piscanec, S.; Jiang, D.; Novoselov, K.S.; Roth, S.; et al. Raman spectrum of graphene and graphene layers. Phys. Rev. Lett. 2006, 97, 187401. [CrossRef] [PubMed]

37. Albrektsen, O.; Eriksen, R.L.; Novikov, S.M.; Schall, D.; Karl, M.; Bozhevolnyi, S.; Simonsen, A.C. High resolution imaging of few-layer graphene. J. Appl. Phys. 2012, 111, 064305. [CrossRef]

38. Graf, D.; Molitor, F.; Ensslin, K.; Stampfer, C.; Jungen, A.; Hierold, C.; Wirtz, L. Spatially resolved raman spectroscopy of singleand few-layer graphene. Nano Lett. 2007, 7, 238-242. [CrossRef] [PubMed]

39. Palik, E.D. Handbook of Optical Constants of Solids; Elsevier: Amsterdam, The Netherlands, 2012; ISBN 9780080547213.

40. Ermolaev, G.A.; Tsapenko, A.P.; Volkov, V.S.; Anisimov, A.S.; Gladush, Y.G.; Nasibulin, A.G. Express determination of thickness and dielectric function of single-walled carbon nanotube films. Appl. Phys. Lett. 2020, 116, 231103. [CrossRef]

41. Zhu, S.-E.; Yuan, S.; Janssen, G.C.A. Optical transmittance of multilayer graphene. EPL 2014, 108, 17007. [CrossRef]

42. Chugh, S.; Man, M.; Chen, Z.; Webb, K.J. Ultra-dark graphene stack metamaterials. Appl. Phys. Lett. 2015, 106, 061102. [CrossRef]

43. Lin, H.; Sturmberg, B.C.P.; Lin, K.-T.; Yang, Y.; Zheng, X.; Chong, T.K.; De Sterke, C.M.; Jia, B. A 90-nm-thick graphene metamaterial for strong and extremely broadband absorption of unpolarized light. Nat. Photonics 2019, 13, 270-276. [CrossRef]

44. Segura, A.; Artús, L.; Cuscó, R.; Taniguchi, T.; Cassabois, G.; Gil, B. Natural optical anisotropy of h-BN: Highest giant birefringence in a bulk crystal through the mid-infrared to ultraviolet range. Phys. Rev. Mater. 2018, 2, 024001. [CrossRef]

45. Yakubovsky, D.I.; Arsenin, A.V.; Stebunov, Y.V.; Fedyanin, D.Y.; Volkov, V.S. Optical constants and structural properties of thin gold films. Opt. Express 2017, 25, 25574-25587. [CrossRef] [PubMed]

46. Wang, Y.; Deng, Z.-L.; Hu, D.; Yuan, J.; Ou, Q.; Qin, F.; Zhang, Y.; Ouyang, X.; Li, Y.; Peng, B.; et al. Atomically thin noble metal dichalcogenides for phase-regulated meta-optics. Nano Lett. 2020, 20, 7811-7818. [CrossRef]

47. Tompkins, H.G.; Irene, E.A. Handbook of Ellipsometry; Springer: Berlin/Heidelberg, Germany, 2005 ; ISBN 9780815514992. 